1 Evaluation of four vertical jump tests: methodology, reliability, validity, and accuracy.

3

4

5

6

7

8 Measurement In Physical Education and Exercise Science, Volume 4,

9 Issue 4, 2000. The original publication is available from their website,

10 http://www.tandfonline.com/doi/abs/10.1207/S15327841MPEE0404_2

11 \#.VRXEyfmG_Cs . It is hereby reproduced according to the ROMEO

12 Green classification of the journal. 
1 Abstract

Vertical jump performance tests can give considerably different

3 results, even when different methods are used to analyze a single jump

4 trial. To evaluate and compare four different methods commonly used to

5 measure vertical jump performance, 52 physically active males each

6 performed five maximal vertical jumps. Kinetic and kinematic data were

7 used to analyze each trial using the four methods: a criterion test based on

8 body center of mass displacement (VJPT); two methods based on vertical

9 take-off velocity as calculated form the force platform (JUMP2 and

10 JUMP3); and one method based on time in the air (JUMPAIR). All four

11 methods showed excellent reliability $(\mathrm{R}>0.97)$. Using VJPT as the

12 criterion, the other three methods showed excellent coefficients of validity

$13(\mathrm{R}>0.95)$ but poor accuracy: the vertical jump results were statistically

14 different among all methods $(\mathrm{p}<0.01)$. From the discussion, JUMPAIR is

15 considered a relatively simple and inexpensive method to obtain valid and

16 reliable measures of vertical jump performance without an arm swing,

17 provided the appropriate adjustments are made to the jump results.

18

19 Key words:

Reliability Validity Vertical jump 
2 Vertical jumping is regarded as an important and attractive element

3 of many sports such as basketball and volleyball. Papers are regularly

4 published in exercise science publications, both lay and scientific, about

5 training methods for vertical jump performance improvement. A key step

6 in any jump training study is vertical jump measurement. Vertical jump

7 tests are also common in Physical Education, Fitness or Sports programs,

8 as a means to assess lower limb "power". However, vertical jump

9 performance results may be considerably different depending on the test

10 used, even when different methods are used to analyze the same jump (H.

11 Hatze, personal communication, November 11, 1992).

12 Traditionally, the most commonly used method is Sargent's test

13 (Sargent's study from 1924, as cited in Johnson \& Nelson, 1974), also

14 known as the jump and reach test (e.g., Blattner \& Noble, 1979; Clutch,

15 Wilton, McGown \& Bryce, 1983; Davies, Greenwood \& Jones, 1988;

16 Genuario \& Dolgener, 1980). This method is simple to use, requiring only

17 a wall or board and chalk powder to make marks with your fingers.

18 Johnson \& Nelson (1974) report a reliability of 0.93 and an objectivity

19 also of 0.93 for this test. Many scientists, however, have resorted to other

20 methods using video systems, landing mats, or force platforms, in order to

21 be able to measure jump height during jumps without an arm swing or

22 under more natural settings, or as an attempt to obtain a higher accuracy or

23 better credibility. The most precise method, the standard hereby called

24 Vertical Jump Performance Test (VJPT), involves calculating the exact

25 position of the body center of mass (BCOM) over time, using

26 cinematography or video techniques. Jump height is obtained by

27 subtracting the position of BCOM when the subject is standing from the 
1 peak BCOM position during flight (Aragón-Vargas, 1997; Bobbert,

2 Huijing \& van Ingen Schenau, 1987; Pandy \& Zajac, 1991). Alternatives

3 include applying particle dynamics equations to calculate take-off velocity

4 of the body and jump height from force plate data (Dowling \& Vamos,

5 1993), or using basic particle kinematics equations to calculate jump

6 height from flight time, as measured by different timing devices

7 (Asmussen \& Bonde-Petersen, 1974; Bosco \& Komi, 1979; Bosco,

8 Luhtanen, \& Komi, 1983; Komi \& Bosco, 1978). Since the necessary

9 equipment is often costly and difficult to use, and given that some of the

10 calculations involve assumptions that are not always acceptable, it is

11 important to know the differences among jump height values obtained

12 using each method.

13 Therefore, the purpose of this paper is to study the reliability of

14 four different methods commonly used to measure vertical jump

15 performance, to calculate the actual test result differences among methods,

16 and to evaluate the ability of each test to predict "true" vertical jump

17 performance, according to the VJPT standard. These are useful

18 quantitative tools for the exercise scientist who wants to compare studies

19 that have used different methodologies, or for the coach, trainer, or

20 physical educator who needs to find out how much more accuracy is

21 obtained by using more costly and sophisticated methods.

22

23 Methods

Fifty-two physically active male college students each performed

26 five maximal vertical jumps, starting from the position of their choice,

27 with their hands on their hips (arms akimbo). All participants gave their 
1 informed consent in accordance with the policy statement of the University

2 of Michigan. They completed three practice jumps before data collection,

3 and were required to wait for one minute after each trial. Participants

4 performed the jumps barefooted, wearing only a swimsuit or pair of shorts.

5 Five reflective markers were placed on the right side of the body, on the

6 glenohumeral joint (shoulder), the greater trochanter (hip), the lateral

7 condyle of the femur (knee), the lateral malleolus (ankle), and the fifth

8 metatarsal (toe). All five trials of all the subjects were used for calculating

9 reliability, but only the best jump (as assessed using equation 1 below) was

10 used for the other comparisons.

11 Ground reaction forces and moments of force were collected with a

12 Bertec force plate (model 4060A), and were sampled at $300 \mathrm{~Hz}$. A video-

13 based $(60 \mathrm{~Hz})$, real-time, 3-D motion analysis system (Motion Analysis

14 Corp.) was used to collect and process kinematic data. Kinematic data were filtered with a low-pass, fourth-order Butterworth filter with an

16 effective cutoff frequency of $8 \mathrm{~Hz}$.

17 Basic anthropometric data were obtained using standard sliding

18 calipers, tape measures, and the force platform. Body mass and body

19 height were measured according to Lohman, Roche, \& Martorell (1988).

20 Thigh length, midthigh circumference, shank length, calf circumference,

21 malleolus width, malleolus height, and foot length were obtained

22 according to Vaughan, Davis, \& O'Connor (1992). These data were used

23 for the calculation of segmental centers of mass (see below).

\section{Data analysis}

The biomechanical model used and all analytic procedures have

26 been described in detail elsewhere (Aragón-Vargas, 1994). Briefly, the

27 human body was modeled as a planar, rigid-body system comprised of 
1 four segments linked by frictionless, hinge joints (figure 1). Kinetic and

2 kinematic data were used to obtain the four different measures of jump

3 height.

4

\section{(Insert figure 1 about here)}

The most accurate method for calculating vertical jump height (VJPT) requires a precise calculation of the body center of mass position throughout the movement from video data. Calculation of the body center of mass position was performed using the method of summation of torques, which in turn requires the calculation of the center of mass position of each segment over time. Segmental centers of mass were calculated according to the procedure of Vaughan et al. (1992), with the exception of HAT, which was calculated according to Aragón-Vargas (1994), based on Clauser, McConville, \& Young (1969), and Hinrichs (1990).

VJPT was obtained directly from the body center of mass (BCOM) position data, by subtracting the vertical position of BCOM while standing from the peak vertical position of BCOM during flight:

$$
V J P T=z B C O M_{p e a k}-z B C O M_{\text {standing }}
$$

VJPT is used in this study as the criterion or standard for comparison. Two alternate methods used in biomechanics for the calculation of jump height require calculating vertical take-off velocity (TOVEL). Take-off velocity was obtained from the instantaneous vertical velocity vs. time curve, which in turn was calculated according to: 
$2 \quad$ Where $F_{z p}$ is propulsive force, obtained from subtracting body

3 weight from the vertical ground reaction force; $t_{0}$ is the beginning of data

4 collection, and $t_{\text {to }}$ is the instant of take-off.

5 Theoretically, jump height depends on both vertical take-off

6 velocity and body center of mass position at take-off (Bobbert \& van Ingen

7 Schenau, 1988), according to the equation:

$$
\mathrm{JUMP} 2=\left[(T O V E L)^{2} *(2 g)^{-1}\right]+z B C O M_{\text {to }}-z B C O M_{\text {standing }}
$$

Equation (3) uses information from both the force platform and the video equipment. Ignoring BCOM elevation before take-off, vertical jump

11 height may be obtained from vertical take-off velocity alone, requiring

12 only force plate data:

$$
\mathrm{JUMP} 3=(T O V E L)^{2} *(2 g)^{-1}
$$
the instant of take-off and the instant of landing. For the sake of this paper, take-off and landing times were obtained from ground reaction force data, when $\mathrm{Fz}<3.0 \mathrm{~N}$ and $\mathrm{Fz}>3.0 \mathrm{~N}$, respectively. Jump height is then obtained using the equation:

$$
\mathrm{JUMPAIR}=g *\left(\frac{\text { tair }}{2}\right)^{2} * 2^{-1}
$$
reliability, that is, the ability of the test to give consistent results. 
1 According to Kerlinger (1988), a correlation coefficient for reliability may

2 be obtained by partitioning the variance obtained from $m$ measurements

3 applied to $n$ subjects, into three components (variance among subjects,

4 variance within subjects, and variance due to error), and obtaining the

5 ratio:

6

7

$$
r_{t t}=\frac{M S S-M S E}{M S S}
$$

8 where MSS is the Subjects Sum of Squares divided by (n-1) and MSE is

9 the Residual (Error) Sum of Squares divided by [(m-1)(n-1)]. (See

10 Kerlinger, 1988; Table 26.2; and equation 26.5).

11 It is possible then to obtain the standard error of measurement

$12(S E M)$ using the group standard deviation $(S D)$ and the reliability of the

13 test $\left(R_{\mathrm{tt}}\right)$ (Baumgartner, 1989):

14

15

$$
S E M=S D \sqrt{1-R_{t t}}
$$

16 which is equal to MSE if calculated according to Kerlinger above.

17 Validity coefficients, regression coefficients, and prediction errors were calculated using simple linear regression techniques, with VJPT as

19 the dependent variable and each of the other methods as the independent

20 variable, according to the general linear model:

$$
y=\beta_{0}+\beta x+\mathrm{E}_{j}
$$

This evaluation of validity is in agreement with common

23 procedures to assess criterion-related evidence of validity (Kerlinger,

24 1988; Wood, 1989). Usually, when such procedures are used, the criterion

25 test and the "new" test have different units of measurement and have been 
1 administered on separate occasions. In this case, however, all results are in

2 meters, and it is possible to evaluate not only the correlation of the

3 measurements (concurrent validity) and the regression coefficients

4 (predictive validity), but also whether all tests provide the same results, a

5 reasonable expectation given that they are all measures of the same

6 performance of the same subjects. This agreement between tests is called

7 accuracy in this paper.

To evaluate the accuracy, average jump heights obtained using

9 each of the four methods were compared using Student's t-test for paired

10 samples, making Bonferroni's adjustment for multiple comparisons, at a

11 significance level of $\mathrm{p}<0.01$. The $95 \%$ family confidence intervals for the

12 difference between VJPT and each of the other three methods were also

13 calculated, using Bonferroni's adjustment for family confidence

14 coefficients.

15

\section{Results}

Table 1 shows basic descriptive statistics for the subjects. Average body weight $(74.3 \mathrm{~kg}$ ) was slightly above the U.S. population average for a body height of $1.79 \mathrm{~m}(71.8 \mathrm{~kg})$ (Metropolitan Life Insurance Company, 1959). Best trial jump heights (VJP) ranged from $0.372 \mathrm{~m}$ to $0.663 \mathrm{~m}$ $($ mean $=0.520 \mathrm{~m})$, and had a coefficient of variation of $13.4 \%$. There were 16 subjects, or $31 \%$ of the sample, outside $\pm 1 S D$ of the average VJP.

(Insert table 1 about here)

Reliability data are presented in Table 2 . The standard or criterion method (VJPT) shows a correlation coefficient of 0.9936 , for a standard error of measurement of $12.7 \mathrm{~mm}$.

(Insert tables 2 and 3 about here) 
1 Table 3 shows descriptive statistics for the jump height results

2 according to each of the four methods used. The highest values were

3 obtained for the standard method, VJPT. The other three methods

4 (JUMP2, JUMP3 and JUMPAIR ) resulted in jump height averages that

5 were $15 \mathrm{~mm}, 159 \mathrm{~mm}$, and $118 \mathrm{~mm}$ lower than VJPT, respectively. These

6 three differences were statistically significant $(\mathrm{p}<0.01)$; furthermore, all

7 the differences between any two methods were statistically significant.

8 Figure 2 presents the $95 \%$ confidence intervals for each difference

9 between VJPT and one of the other three methods. This figure graphically

10 shows the underestimation of jump height normally obtained from using

11 each of those methods.

12

(Insert figure 2 about here)

14 Table 4 shows the simple regression analysis results.. Coefficients

15 of correlation $(R)$ represent the validity of each method, using VJPT as the

16 criterion or previously validated test. All methods were able to explain

17 more than $90 \%$ of the vertical jump height variability (see column for $R^{2}$ ).

18 The estimated prediction error of all three methods is close to $20 \mathrm{~mm}$.

19 Lastly, it must be pointed out that the $\beta$ coefficients for models 2 and 3 are

20 very close to 1.0 , and therefore the $\beta_{0}$ constant is similar to the jump

21 height average differences indicated above.

$22 \quad$ (Insert table 4 about here)

23 Discussion

Reliability

Before making any meaningful comparisons among jump test

26 methods, it is necessary to have a good standard or criterion. In this

27 particular case, VJPT had been chosen as the standard based on theoretical

28 arguments. This test shows an excellent reliability and a small SEM (see 
1 Table 2). VJPT was compared with three other methods for testing vertical

2 jump height, but not with the one most commonly used, Sargent's jump-

3 and-reach test. Sargent's test involves an arm swing during the propulsion

4 phase, and this additional variable precludes any meaningful comparisons

5 from being made ${ }^{1}$.

6 Under normal testing circumstances, variability in the results

7 comes from two major sources: "true" variability, showing differences in

8 performance both between and within subjects, and the error introduced by

9 the measuring method. The four variables presented in tables 2 and 3 are

10 measures of the same vertical jump performance. Therefore, "true"

11 variability (in this case only from differences between subjects, as only the

12 best trial was used for the analysis) must be the same for all methods, and

13 it is then possible to use VJPT as the standard to compare both the

14 absolute $(S D)$ and relative (Coefficient of Variance, $C V$ ) variability (Table

153 ) introduced by the other methods. Relative variability is higher for all

16 three methods compared to VJPT.

17 Additional absolute variability is only introduced by JUMP2 $(\triangle S D$

$18=7 \mathrm{~cm}$ ), while JUMP3 and JUMPAIR show smaller values than VJPT. An

19 instrument or method may show less variability because it is less sensitive

20 and does not discriminate so clearly among different performances, or

21 because it really has a smaller error of measurement. Using this

22 information together with the reliability coefficients and SEM values

23 (Table 2), it is clear that VJPT and JUMPAIR are the most stable,

24 consistent measures of the true vertical jump, while JUMP2 is the least 25 consistent. 
1 While validity coefficients and prediction errors obtained are

2 excellent, it is clear that all four vertical jump test methods give different

3 results, i.e., the three alternative methods are not accurate. JUMP2 is

4 theoretically correct, but it requires a perfect synchronization between the

5 force plate and video signals. A synchronization error will cause the test

6 administrator to use a take-off position (obtained from video data) which

7 does not correspond to the same instant of the take-off velocity (obtained

8 from the force platform). An error of only $16.7 \mathrm{~ms}$ (one frame at a

9 sampling rate of $60 \mathrm{~Hz}$ ) would result in under- or over-estimating the

10 relative position of take-off (and therefore jump height) by $44 \mathrm{~mm}$

11 (Aragón Vargas, 1994).

12 The calculation of JUMP3 involves only force-plate data, and

13 therefore has no signal synchronization problems. On the other hand, it

14 does not take into account the relative take-off height of the subject. This

15 should not pose any problem, since previous studies have shown that the

16 major contribution to vertical jump height differences among subjects

17 comes from take-off velocity (TOVEL), while the relative take-off height

18 is very similar from one subject to another (Aragón-Vargas, 1997). The

$1995 \%$ confidence interval for relative take-off height in the present study

20 was $14.4 \pm 0.73 \mathrm{~cm}$ which, according to equations (1), (3), and (4), should

21 agree with the difference between JUMP3 and VJPT. The 95\% confidence

22 interval for the difference was $15.9 \pm 0.7 \mathrm{~cm}$, showing a discrepancy

23 between VJPT and "corrected" JUMP3 of $15 \mathrm{~mm}$ that we are unable to

24 account for.

25 Lastly, the calculation of jump height using the method JUMPAIR

26 has been criticized in the literature because some of the assumptions

27 involved are not correct (Dowling \& Vamos, 1993; H. Hatze, personal 
1 communication, November 11, 1992). One clear limitation is that equation

2 (5) assumes that the time the center of mass of the body is falling is equal

3 to one-half of the time in the air. In other words, the time that BCOM

4 travels upwards should be equal to the time it travels downwards, which is

5 only true if the subject takes off and lands with his body in the same

6 position. In the present study, the time down was significantly longer than

7 time up (average difference $=0.016 \mathrm{~s}, \mathrm{p}<0.0001$ ), suggesting the subjects

8 landed with their bodies partially crouched ${ }^{2}$. This results in an

9 overestimation of the distance from take-off to peak, as may be seen

10 comparing JUMPAIR with JUMP3, a method that does not consider

11 relative take-off height either (see Table 3). The final result, however, is

12 lower than VJPT.

Practical recommendations.

14 Results from Tables 2 to 4, and figure 2, provide the necessary

15 information for choosing from the three alternative methods for predicting

16 true jump height, as measured by VJPT. All three methods have excellent

17 reliability, an essential first step. Validity coefficients are also excellent for

18 all three tests. JUMP2 gives the smallest average difference in jump

19 height, but regression analysis (cf. Table 4) shows that the estimation error

20 is larger for this method. Furthermore, since its slope ( $\beta$ coefficient) is

21 significantly different from 1.0, the estimation error will vary with the

22 level of the results, underestimating true jump height for some subjects,

23 and overestimating it for others. JUMP3 and JUMPAIR show larger

24 average differences with the criterion test, but the differences are more

25 stable, independent of the level of the results, and the prediction error is

26 smaller. 
1 Most vertical jump performance studies seek to compare jump

2 height before and after a particular treatment (a training program). For this

3 type of comparison, it is not really important if different methods give

4 different results, provided the same method is used for the pre- and post-

5 tests, and provided the method used shows good reliability and validity

6 coefficients, as is the case for all the three methods evaluated in this

7 study $^{3}$. If the investigator or coach is more interested in being able to

8 compare results obtained with different methods, it is clear that

9 comparisons will be meaningless unless the differences inherent to each

10 method are considered. The parameters presented in this study will allow

11 making the necessary adjustments to achieve a reasonable degree of

12 accuracy.

13 Considering all the criteria above, and taking into account the

14 equipment necessary for testing according to each method, the most simple

15 and less expensive method is the one that calculates jump height from time

16 in the air, using a landing mat and a timer. Time in the air may also be

17 obtained from force plate data, as in the present study. According to the

18 present data, very little reliability and validity is compromised, and the

19 results may be used to calculate true jump height with confidence. 


\section{Acknowledgments:}

This research project was made possible by a Rackham

3 Dissertation Grant and a Rackham Predoctoral Fellowship from the

4 University of Michigan, and Grant VI-245-95-276, School of Physical

5 Education, University of Costa Rica.

6 I appreciate the valuable support from our School Director,

7 Wilfridio Mathieu, M.Sc. and the assistance of Cinthya Campos, M.Sc.

8 during the completion of the project. Special thanks to Dr. Walter Salazar

9 for his input on the manuscript. Data collection was possible thanks to Dr.

10 M. Melissa Gross, Human Movement Research Center, Division of

11 Kinesiology, The University of Michigan.

12 


\section{References}

2 Aragón-Vargas, L. F. (1994). Kinesiological limits of vertical jump performance. Unpublished doctoral dissertation, The University of Michigan, Ann Arbor.

5 Aragón-Vargas, L. F. (1996). Comparación de cuatro métodos para la medición del salto vertical [Comparison of four methods for measurement of the vertical jump]. Revista Educación 20(1), 3340.

Aragón-Vargas, L. F. (1997). Kinesiological factors in vertical jump performance: differences among individuals. Journal of Applied

Biomechanics 13(1), 24-44

Asmussen, E., \& Bonde-Petersen, F. (1974). Storage of elastic energy in skeletal muscles in man. Acta Physiologica Scandinavica, 91(3), 385-92.

Baumgartner, T. A. (1989). Norm-referenced measurement: reliability. In Safrit, M. J., \& Wood, T. M. (Eds.), Measurement Concepts in

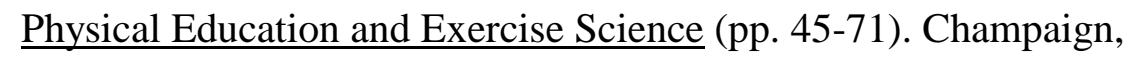
Quarterly, 50(4), 583-588. 
1 Bobbert, M. F., Huijing, P. A., \& van Ingen Schenau, G. J. (1987). Drop jumping I. The influence of jumping technique on the biomechanics of jumping. Medicine and Science in Sports and Exercise, 19(4), 332-338.

Bobbert, M. F., \& van Ingen Schenau, G. J. (1988). Coordination in vertical jumping. Journal of Biomechanics, 21(3), 249-262.

Bosco, C., \& Komi, P. V. (1979). Mechanical characteristics and fiber composition of human leg extensor muscles. European Journal of Applied Physiology, 41, 275-284.

Bosco, C., Luhtanen, P., \& Komi, P. V. (1983). A simple method for measurement of mechanical power in jumping. European Journal of Applied Physiology, 50(2), 273-82.

Clauser, C. E., McConville, J. T., \& Young, J. W. (1969). Weight, volume, and center of mass of segments of the human body. Wright-Patterson Air Force Base, Ohio.

Clutch, D., Wilton, M., McGown, C., \& Bryce, G. R. (1983). The effect of depth jumps and weight training on leg strength and vertical jump. Research Quarterly for Exercise and Sport, 54, 5-10.

Davies, B. N., Greenwood, E. J., \& Jones, S. R. (1988). Gender difference in the relationship of performance in the handgrip and standing long jump tests to lean limb volume in young adults. European Journal of Applied Physiology, 58(3), 315-320. 
1 Dowling, J. J., \& Vamos, L. (1993). Identification of kinetic and temporal

2 factors related to vertical jump performance. Journal of Applied Biomechanics, 9, 95-110.

Genuario, S. E., \& Dolgener, F. A. (1980). The relationship of isokinetic torque at two speeds to the vertical jump. Research Quarterly for Exercise and Sport, 51(4), 593-598.

Hinrichs, R. N. (1990). Adjustments to the segment center of mass proportions of Clauser et al. (1969). Journal of Biomechanics, 23(9), 949-951.

Johnson, B. L. \& Nelson, J. K. (1974). Practical measurements for evaluation in physical education (2nd ed.). Minneapolis, MN: Burgess Publishing Co.

Kerlinger, F. N. (1988). Investigación del comportamiento [Foundations of behavioral research] (2nd ed.). Mexico: McGraw-Hill.

Komi, P. V., \& Bosco, C. (1978). Utilization of stored elastic energy in leg extensor muscles by men and women. Medicine and Science in Sports, 10(4), 261-265.

Lohman, T. G., Roche A. F., \& Martorell, R. (1988). Anthropometric standardization reference manual. Champaign, IL: Human Kinetics.

Metropolitan Life Insurance Company (1959). Actuarial tables. New York: Metropolitan Life Insurance Company. 
1 Pandy, M. G., \& Zajac, F. E. (1991). Optimal muscular coordination

2

3 Vaughan, C. L., Davis, B. L., \& O'Connor, J. C. (1992). Dynamics of

4 strategies for jumping. Journal of Biomechanics, 24(1), 1-10. human gait. Champaign, IL: Human Kinetics.

Wood, T.M. (1989). The Changing Nature of Norm-Referenced Validity. In Safrit, M. J., \& Wood, T. M. (Eds.), Measurement Concepts in Physical Education and Exercise Science (pp. 23-44). Champaign, IL: Human Kinetics.

\section{Notes}

${ }^{1}$ A separate study using the Sargent jump test with 56 subjects performing five jumps each, showed a reliability correlation coefficient of 0.9859 , which is still very good. (Unpublished data).

${ }^{2}$ Apparently, this situation is worsened when using an arm swing. A separate study (mentioned in footnote \#1) has shown the reliability of JUMPAIR to decrease under these circumstances, to 0.9558 , which may be partially accounted for by the variation in the position of the arms at takeoff and landing (Unpublished data).

${ }^{3}$ This practical application assumes that the reliability obtained in this study when trials are separated by only a few minutes can be extrapolated to a study when trials are separated by several weeks or months. 


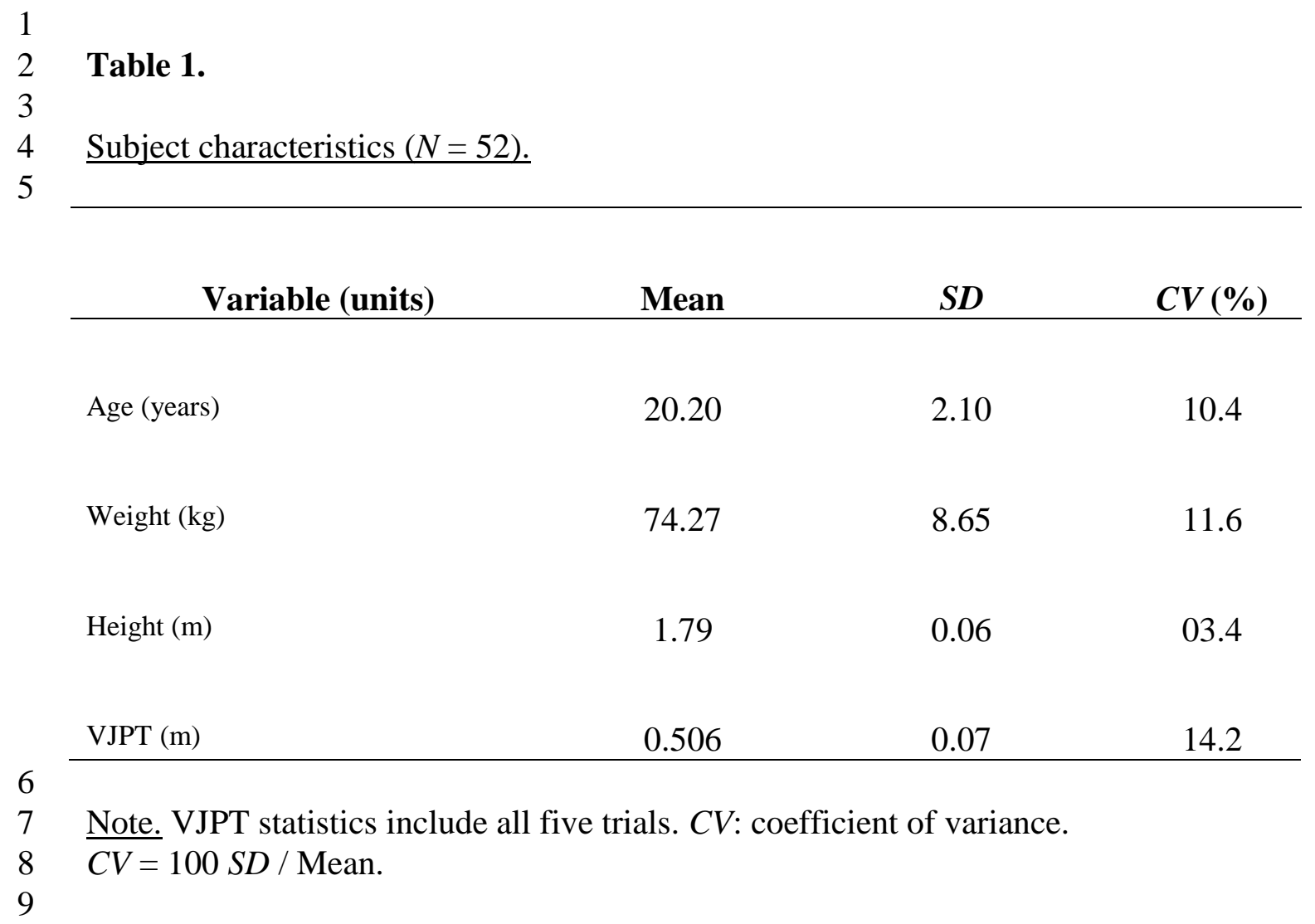


3 Table 2.

4

$5 \quad$ Reliability calculations for four jump tests. $(N=49, \mathrm{i}=5)$.

6

\begin{tabular}{lcccc}
\hline & VJPT & JUMP2 & JUMP3 & JUMPAIR \\
\hline$R_{\mathrm{tt}}$ & 0.9936 & 0.9704 & 0.9859 & 0.9936 \\
$\left(R_{\mathrm{tt}}\right)^{2}$ & 0.9873 & 0.9417 & 0.9719 & 0.9872 \\
& & & & \\
$S E M(\mathrm{~mm})$ & 12.7 & 27.8 & 18.0 & 12.1 \\
\hline
\end{tabular}

$7 \quad$ Note. $R_{\mathrm{tt}}$ is the reliability correlation coefficient; $\left(R_{\mathrm{tt}}\right)^{2}$ is the reliability

8 coefficient of determination.

9 
1

3 Table 3.

4

5 Descriptive statistics for each jump height method $(N=52)$.

\begin{tabular}{lcccc} 
& VJPT & JUMP2 & JUMP3 & JUMPAIR \\
\hline & $.520^{\mathbf{a}}$ & $.505^{\mathbf{a}}$ & $.361^{\mathbf{a}}$ & $.402^{\mathbf{a}}$ \\
Average (m) & .372 & .365 & .240 & .263 \\
Minimum (m) & .663 & .667 & .503 & .550 \\
Maximum (m) & .070 & .077 & .066 & .067 \\
$S D(\mathrm{~m})$ & & & & \\
& & & & \\
$C V(\%)$ & 13.4 & 15.3 & 18.3 & 16.6 \\
\hline
\end{tabular}

$6 \quad$ Note. The best trial from each subject was used in the calculations. From

7 "Comparación de cuatro métodos para la medición del salto vertical", by L.F.

8 Aragón-Vargas, 1996, Revista Educación, 20(1), p. 37. Copyright 1996 by the

9 Editorial de la Universidad de Costa Rica. Reprinted with permission.

10 a) All mean differences are statistically significant, $\mathrm{p}<0.01$.

11

12

13

14 


\section{Table 4.}

4

5 Simple regression analysis $(N=52)$.

6

\begin{tabular}{|c|c|c|c|c|}
\hline Model & $\boldsymbol{R}$ & $R^{2}$ & MSE & Error \\
\hline 1) $\mathrm{VJPT}=0.087+0.857^{\mathbf{a}} \mathrm{JUMP} 2$ & .952 & .906 & $.464 \mathrm{E}-03$ & .0215 \\
\hline 2) $\mathrm{VJPT}=0.154+1.014 \mathbf{b} \mathrm{JUMP} 3$ & .961 & .924 & $.376 \mathrm{E}-03$ & .0194 \\
\hline 3) $\mathrm{VJPT}=0.117+1.002^{\mathbf{b}}$ JUMPAIR & .962 & .926 & $.369 \mathrm{E}-03$ & .0192 \\
\hline
\end{tabular}

7 Note. Only the best trial was used in the analysis. All statistical models are

8 significant $(\mathrm{p}<0.0001)$. From "Comparación de cuatro métodos para la

9 medición del salto vertical", by L.F. Aragón-Vargas, 1996, Revista Educación,

$10 \underline{20(1)}$, p. 38. Copyright 1996 by the Editorial de la Universidad de Costa Rica.

11 Reprinted with permission.

12 a) This coefficient is significantly different from $1.0,(\mathrm{p}<0.01)$.

13 b) These coefficients are NOT significantly different from 1.0, $(\mathrm{p}>0.01)$.

14

15

16 


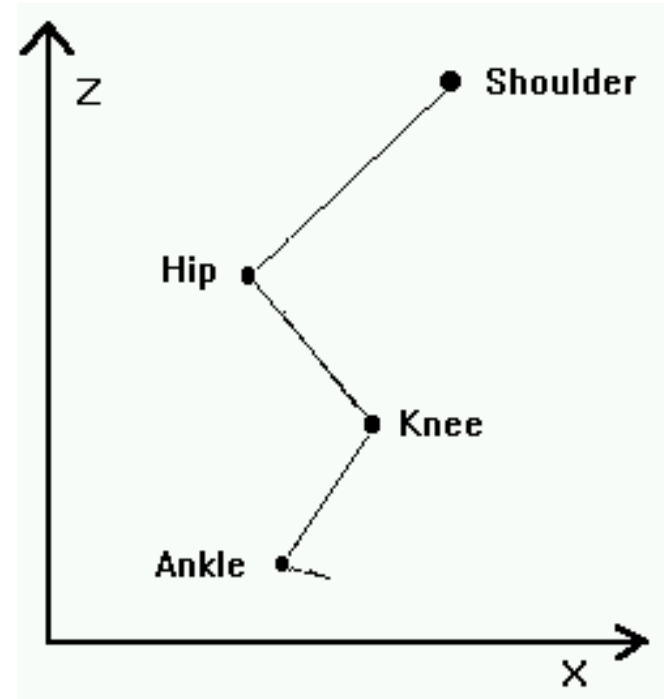

3

4

Figure 1. Biomechanical model. Segments $(i=1$ to 4) are defined by the markers: segment 1 , head, arms and trunk (HAT), from shoulder to hip; segment 2, thighs (THI), from hip to knee; segment 3, shanks (SHA), from knee to ankle, and segment 4, feet (FET), from ankle to toe. 
1

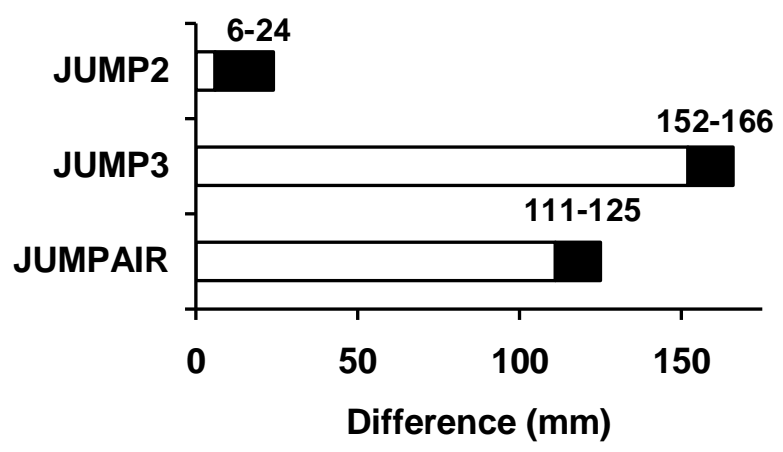

2

Figure 2: $95 \%$ confidence intervals for the difference between the standard method and each one of the other methods (best trial only, $\underline{N}=52$ ). From "Comparación de cuatro métodos para la medición del salto vertical", by L.F. AragónVargas, 1996, Revista Educación, 20(1), p. 37. Copyright 1996 by the Editorial de la Universidad de Costa Rica. Reprinted with permission. 